\title{
A Study on the Equalization of Basic Public Services in the Process of Urban and Rural Integration
}

\author{
Xian Zhang \\ College of Economics and Management, Yunnan Agricultural University, Kunming City, Yunnan Province, \\ 650201, China
}

Email: 280235662@qq.com

Keywords: Urban and rural integration, Basic public service, Equalization, Connotation, Countermeasures

\begin{abstract}
In the vast rural areas, the government invested in compulsory education, medical health, infrastructure construction and other fields inadequately, which is the main factor to form the gap between urban and rural areas. In turn, it restricts the social, economic and cultural development of the countryside and forms a vicious circle of development. Therefore, it is of great practical significance to promote the equalization of basic public services in urban and rural areas. Based on the author's learning and practical experience, this paper first studied the connotation of equalization of basic public services, then analyzed the main causes that restrict the equalization of basic public services in urban and rural areas, and finally put forward the countermeasures to gradually achieve equalization of basic public services in urban and rural areas.
\end{abstract}

\section{Introduction}

Due to the long-term influence of two dimensional structures in urban and rural areas, there are great differences in the supply of basic public services between urban and rural areas in China, forming a relatively independent and fragmented basic public service supply system in urban and rural areas. There is a serious imbalance in the supply and efficiency of basic public services in urban and rural areas, and the gap between urban and rural development is widened. This has caused great bottleneck effect to the progress of urban and rural integration in China and even the healthy and sustained development of the whole national economy. Promoting the equalization of basic public services in urban and rural areas, accelerating the integration of urban and rural development, solving the two dimensional pattern of urban and rural areas, narrowing the gap between urban and rural basic public services and enabling our farmers to share the dividends of reform and development are the responsibilities of the government and the rights of citizens.

\section{The Connotation of the Equalization of Basic Public Services}

Group of government public service specially established by China Administration Association gave the definition of public service, which means to provide public products and services, including the strengthening of urban and rural public infrastructure construction, the development of social employment, social security, education, science and technology, culture, health and other public service and release of public information and so on. Public service is a product and service that is provided by the government to exercise its functions, including value judgments for the public's common enjoyment and to meet social public needs and achieve social equity. Due to the balance of all public services can not be met at the same time, the basic public service makes the limited scope according to the principle of importance and basis within a period of time, which is the concept of the basic public service. The basic fields and basic aspects are two important attributes. It needs to be pointed out that the scope of basic public services is the product of a country's political decision making (political game) in a specific historical period, which is compatible with the corresponding economic and social development stages, and also expands with the development of economy and society. The author believed that basic public services should 
cover basic education, infrastructure, medical and health, social security, employment and other basic public goods and services are related to the livelihood of the people.

The equalization of basic public services can be simply defined as: all residents in different regions in a country can enjoy basically the same basic public services. It contains two aspects: first, the equal content is the basic public service; second, the equality of the basic public service is relative. At present, the equalization of basic public services proposed by our country is lower level equalization based on the guaranteeing the "basic level" equalization. The following principles should be grasped in our country to realize the equalization of urban and rural basic public services: first, we should focus on the realization of the equalization of basic public services in rural and urban areas in rural areas; the second is to avoid the "averaging" of the wrong tendency to grasp the relativity; the third is to recognize that the equalization of basic public services between urban and rural areas is a long-term dynamic process, which is gradually realized along with the development of social economy and the improvement of institutional system.

\section{The Main Source of Restricting the Equalization of Basic Public Services in Urban and Rural Areas}

\subsection{Imperfect public financial system}

The imbalance between national finance and investment between urban and rural areas is an important factor in the imbalance of urban and rural development in China. In 1994, the tax sharing financial system reform greatly enhanced the central government's financial capacity, but it did not invest enough in local governments, and it also weakened the local government's financial capacity. The contradictions like financial power moving up, management power moving down and financial and management power among governments cause that the local financial burden is too heavy and the supply capacity of basic public service is insufficient. This often leads to the government's selective provision of basic public services and phenomenon of "heavy cities and light rural", resulting in the inequality of basic public services between urban and rural areas.

\subsection{The supply mechanism of basic public service in urban and rural areas is not perfect}

Our basic public services have been mainly invested and paid by the government for a long time, and the government has covered production and distribution. The government integrates public service decision-makers, providers and supervisors. Due to the inaccuracy of the role of the government, sometimes there is an "all in the way" government with an "offside" phenomenon. Sometimes there is an "absence" phenomenon in the absence of government responsibility. The supply of basic public services should adhere to the leading role of the government, but this does not mean that the government needs to arrange the supply of all public services, nor does it mean that the government can shirk its due responsibilities. Lacking the diversified public service supply mode, the government has undertaken too many things that can be allowed to do by the market, which is prone to appear government's "offside" tendency, and insufficient supply of basic public services in rural areas. It is easy for government responsibility to fail to perform; while, the absence of government functions is present.

\subsection{The standard of government performance assessment is not perfect, and the performance of government public service function is not in place}

Since the reform and opening to the outside world, China's economic growth has been rapid, which has a great relationship with the growth of GDP as a local official's performance assessment standard. This also leads to the local government's selective performance of the government's functions, emphasizing on economic development, and the lack of attention to the basic public services in the countryside and the indifference of consciousness. Moreover, the function of the government in the supply of basic public service in rural areas is not in place. Most of the supplies of rural public services, such as health care, public culture, infrastructure are mostly carried out the "top-down" supply path, which deviates from the actual needs of rural residents for public services. 
Lacking the necessary right to speak in the demand for basic public services will cause that the contradiction between supply and demand is not consistent, and the "dislocation" and "absent" of government in the supply of rural basic public services on their functions, which exacerbate the non equalization of basic public services in urban and rural areas.

\section{The Path to Gradually Realize the Equalization of Basic Public Services in Urban and Rural Areas}

\subsection{Strengthening the reform of the household registration system}

Promoting the reform of the household registration system is a key measure to promote the urbanization of the people and gradually realize the equalization of basic public services in urban and rural areas. We should continue to strengthen the reform of household registration system, reduce the threshold of integrating household into a small and medium-sized city, relax the conditions of settlement, establish a unified household registration system in urban and rural areas, and gradually abolish the distinction between agricultural household registration and non agricultural household registration, so that to gradually achieve the integrated management of urban and rural household registration. Promoting the integration of urban and rural basic public services and standardized development, can make farmers and citizens enjoy equal benefits in education, health care, employment, housing, social security and other basic public services such as "national treatment", and form a new pattern of integrated urban and rural socio-economic development. The system of urban and rural integration should be used to ensure the equalization of basic public services, reduce the burden on farmers and improve the quality of life of farmers. We should improve the compensation mechanism for the land expropriated farmers, as well as the basic public service guarantee after their land loss, and solve the worries of the landless farmers. Further more, we should promote rural residents to integrate into the process of urban and rural integration, improve the happiness of rural residents instead of passively involved in the wave of urban rural integration, so as to truly achieve the people-oriented integration of urban and rural development.

\subsection{Improve the public financial system of equalization of basic public services}

We should improve the public finance system of equalization of basic public services, optimize the structure of public expenditure, increase the input of public finance to basic public services, and broaden the beneficial side of fiscal revenue and expenditure, especially in rural areas, backward areas and vulnerable groups. Moreover, we should improve the transfer payment system, increase the scale and proportion of the general transfer payment, especially increase the transfer payment to the less developed areas and the rural areas, and at the same time, reduce the financial absorption to the less developed areas. We should standardize the special transfer payment to improve the local government's financial security ability in the process of promoting the equalization of basic public services in urban and rural areas. At the same time, we should enhance the transparency of fiscal expenditure for basic public services, increase the tracking and supervision of fund utilization, and give full play to the positive role of public finance in promoting the equalization of basic public services in urban and rural areas.

\subsection{Innovate the basic public service supply model and introduce multiple supply mechanism}

The government can provide basic public services in a variety of ways, such as purchase, contract entrustment, franchising, government participation, and service outsourcing. Mobilizing the enthusiasm of social forces to participate in the supply of basic public services, not only can save public expenditure, make up for the financial resources of the government or take care of and the insufficient supply of rural basic public services issues, but also can improve the efficiency and quality of urban and rural basic public services to provide practical and diverse basic public service for rural residents. In the process of promoting the socialization and marketization of basic public services in urban and rural areas, we should encourage rural residents to participate in the supply of basic public services in rural areas of decision process and pay attention to "the voice of the people", 
so that to play the role of government and market in promoting the equalization of basic public services in the process of "double wheel drive".

\subsection{Establish performance evaluation and supervision mechanism for the equalization of basic public services in urban and rural areas}

The first is to establish a performance evaluation system for the equalization of basic public services in urban and rural areas. The equalization of basic public service evaluation projects should include compulsory education, medical and health, employment services, housing security, public culture and other fields. On the main body of evaluation, we should get rid of the single subject of the government, take the citizen as the basic receiver of the public service as the center, and enhance the citizens' right to speak in the basic public service. We need to take citizen satisfaction as an important yardstick of evaluation. The introduction of the third party assessment will make the performance evaluation of urban and rural basic public services more objective and fair. We should apply multiple performance evaluation systems combined with various evaluation subjects, which can effectively change the way of basic public service supply; and this is "responsible for the whole and responsible for the people". The second is to improve the supervision mechanism. In order to improve the efficiency and fairness of urban and rural basic public service supply, we must establish effective urban and rural basic public service resource allocation and service project purchase process to introduce social supervision mechanism, and form a multi-level, multi-agent participation in the modern regulatory system. When the market mechanism is introduced to the supply of public services, the government should not let the government let it out, but should strengthen the responsibility of the government. The government should track and supervise all public sectors and units in the whole process of decision-making, execution, capital use and final performance.

\section{Acknowledgements}

National Social Science Fund Project: A study on the demand and supply of villagers' public culture in the process of urban and rural integration in the western border ethnic areas (14BMZ107).

\section{References}

[1] Liu Guojun. Realizing the Equalization of Basic Public Services and the Integrated Development of Urban and Rural Areas in Guangdong [J]. Southern Journal, 2009, 02:12-15.

[2] Wen Min, Wen Bo. A Literature Review of the Research on the Equalization of Basic Public Services at Home and Abroad [J]. Journal of Zhaotong College, 2016, 03:73-78+89.

[3] Yang Xi, Wang Ping. Research on the Equalization of Basic Public Services in Urban and Rural Areas from the Perspective of Urban and Rural Integration [J]. Journal of Changchun University of Science and Technology (Social Science Edition), 2017, 01:17-21+62.

[4] Du Yingying. Research on the Reform of the Equalization of Basic Public Services in Tongling City, Tongling [J]. Small and Medium-sized Enterprise Management and Technology (Last Journal), 2015, 01:136-137.

[5] Shen Yuxin, Hua Yanling. Research on the Equalization of Basic Public Services in Rural Areas of Nanjing [J]. Rural Economy in Jiangsu, 2015, 04:28-29.

[6] Liu Yichen, Liu Yiqing. Research on the Equalization of Basic Public Services in Urban and Rural Areas [J]. Cooperative Economy and Science and Technology, 2015, 22:178-179.

[7] Bo Aijing. The Effect and Mechanism of the Equalization of Urban and Rural Basic Services [J]. Journal of Zhengzhou Railway Vocational and Technical College, 2011, 03:82-84. 